\title{
Estimating Parameter of Nonlinear Bias Correction Method using NSGA-II in Daily Precipitation Data
}

\author{
Angga Wahyu Pratama*, Agus Buono, Rahmat Hidayat, Hastuadi Harsa \\ Bogor Agricultural University, Jl. Raya Darmaga, Dramaga Campus, Bogor 16680, Indonesia \\ *Corresponding author, e-mail: angga_jisung13@apps.ipb.ac.id, agusbuono@apps.ipb.ac.id, \\ rahmat.hidayat.asad@gmail.com, hastuadi@gmail.com
}

\begin{abstract}
Nonlinear (NL) method is the most effective bias correction method for correcting statistical bias when observation precipitation data can not be approximated using gamma distribution. Since NL method only adjusts mean and variance, it does not perform well in handling bias on quantile values. This paperpresents a scheme of NL method with additional condition aiming to mitigate bias on quantile values. Non-dominated Sorting Genetic Algorithm II (NSGA-II) was applied to estimate parameter of NL method. Furthermore, to investigate suitability of application of NSGA-Il, we performed Single Objective Genetic Algorithm (SOGA) as a comparison. The experiment results revealed NSGA-Il was suitable when solution of SOGA produced low fitness. Application of NSGA-II could minimize impact of daily bias correction on monthly precipitation. The proposed scheme successfully reduced biases on mean, variance, first and second quantile However, biases on third and fourth moment could not be handled robustly while biases on third quantile only reduced during dry months.
\end{abstract}

Keywords: java island, chirps, daily precipitation, multi-objective, genetic algorithm

Copyright $\odot 2018$ Universitas Ahmad Dahlan. All rights reserved.

\section{Introduction}

Generally, most of Satellite-only Precipitation Products (SPPs) have significant biases. Hence, for further analysis, they require post processing of bias correction [1-3]. Climate Hazards Group InfraRed Precipitation with Stations (CHIRPS) is produced by blending station observations with satellite-only Climate Hazards Group InfraRed Precipitation (CHIRP) which is developed to support drought monitoring [4]. Blending process is supposed to reduce bias of CHIRP. However, a study which was conducted [5] over Cyprus revealed that the mean difference between CHIRPS and observation increased over time. Tote et al [6] showed that CHIRPS had higher bias during dry season. However, study based on CHIRPS in Indonesia is limited. Once, preliminary study over Java Island had been conducted, and it was found that statistical biases of CHIRPS was high during wet season. Based on the previous result, applying bias correction to product low biases precipitation data which outperforms CHIRPS especially during wet season is interesting to be performed. Furthermore, it is crucial to determine suitable bias correction method based on bias indicators to correct.

In recent years, several bias correction methods have been introduced, including meanbased and distribution based. [2, 7, 8] conducted studies to compare several bias correction methods on daily SPPs. Mean-based methods outperformed distribution-based on reducing biases on time series-based indicators (Nash-Sutcliffe coefficient, correlation) [8]. Distributionbased methods was the most effective at handling frequency-based indicators (standard deviation, percentile) [2, 8]. Quantile mapping based on Gamma distribution (QMG) [9] was the most suitable method on mitigating frequency-based bias indicators since it offeredthe best combination of accuracy and robustness. However, QMG only produced valid result when precipitation data can be approximated using gamma-distribution [7]. If the assumption is not met, non linear (NL) [10] correction method was the most effective. QMG method is utilized to match the distribution function of SPPs data to observations. It is used to adjust mean, standard deviation and quantiles [8]. On the other hand, NL method adjusts mean and standard deviation of SPPs data to observation. Unfortunately, discussions over theimpact of NL correction on quantiles values are limited on literature. Since NL method only adjusts mean and standard deviation, it is possible to product corrected data with different quantiles values from 
observations. However, significant difference on quantiles values can lead to different distribution. On that premise, NL method requires additional condition to mitigate biases on quantiles values.

In this paper, the issue of limitations of CHIRPS during wet season over Java Island is expected to be addressed by appplying NL method to correct statistical biases on satellite-only CHIRP using observed precipitation data from Meteorological Climatological and Geophysics Agency (BMKG) as the reference data. A scheme of NL bias correction with additional condition aiming to mitigate biases on quantile values is proposed in this research. The additional conditions are to add thresholds to NL method while most of study which used NL method only working with a threshold [8, 11]. Genetic Algorithm (GA) is utilized to provide parameter prefactor and scaling exponent of power-law function in NL method, the objectives are to achieve minimum difference both mean and coefficient of variance (CV) of corrected CHIRP to observation data. [12] used both single objective and multi objective genetic algorithm to optimize the multivariable sliding mode PI control parameters for process control. The results revealed analyzing the Pareto front of multi objective genetic algorithm increased the control efficiency and needs lower control input than obtained by single objective genetic algorithm. Therefore, in this paper we apply both Multi objective genetic algorithm (MOGA) Non-dominated Sorting Genetic Algorithm II (NSGA - II) [13] and Single Objective Genetic Algorithm (SOGA). Since the optimization of NL method uses two objectives, we combine the two objectives so that SOGA is able to applied.

The literature of application of GA in NL bias correction method is limited. Therefore, preliminary study to determine search space is required before applying GA. In this reseach, point-to-pixel correction is performed, bias correction only applies to pixels of CHIRP corresponding to observation stations. Furthermore, the scheme of bias correction is applied on different months to mitigate high variability of daily precipitation data. Later, the results are evaluated to observe their statistical moment values. In short, the current study specifically aims to determine: (1) whether NSGA-II with its Pareto solution concept is suitable for NL bias correction method, and (2) whether the proposed scheme successfully reduces biases of $\mathrm{CHIRP}$ on statistical moments and quantiles values.

\section{Methodology}

Figure 1 shows the flow charts used in this research. Observations data used in this research are from six BMKG stations, including Pondok Betung, Karang Ploso, Bandung, Tegal, Sangkapura, Banyuwangi. The first two mentioned stations are used as training data for constructing and testing performance of the proposed scheme and search space before applying them to the last four mentioned stations. Optimal GA (SOGA and NSGA-II) parameters, including probability crossover (pc), probability mutation (pm), population size, and maximum generation are generated from training process using January precipitation data, Pondok Betung Station. Temporal resolution of data is daily-type data, from January 2005 to December 2016.

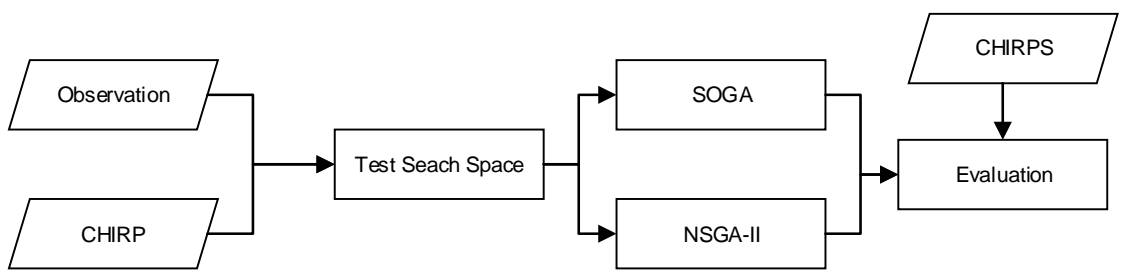

Figure 1. Research Method

\subsection{Proposed Scheme and Search Space of Genetic Algorithm}

The Proposed scheme in this trial is to add thresholds on NL method. Adopted from [8], initially, to handle many days with no precipitation, adding equation for certain threshold is applied in running the proposed schme. Second threshold is constructed to lower precipitation 
values around second quantile of CHIRP since second quantile of CHIRP overestimates observation for most of all months. Morever, last threshold is constructed to escalate precipitation values since CHIRP cannot predict extreme values of observation data well. Search space is determined based on the objective of each thresholds. Search space is constructed by using observation and CHIRP data corresponding to Pondok Betungstation, and the data is obtained from January precipitation data. Furthermore, it is also tested using January and July precipitation data from Karang Ploso station.SOGA with objective function to minimize difference in variance value of corrected data to observation data is used to construct and test search space. The result fromsearch space is utilized to apply bias correction onthe other four stations using GA (SOGA and NSGA-II).

\subsection{Single-objective Genetic Algorithm}

The objective of performing Single-objective Genetic Algorithm (SOGA) is to verify whether the multiobjective problem on estimating parameter of NL method has to be solved using multiobjective optimization. The objectives on estimating parameter of NL method are to minimize the difference in both mean and CV of corrected data to observation data [2, 7, 8].Objective function of SOGAin this study is a combination of the objectives. As a replacement of $\mathrm{CV}$, we use variance $\left(S^{2}\right)$ and combine it with mean $(\bar{x})$ to construct objective function. Objective function of SOGA is shown in Equation 1. Equation 1 is invers of the objective. Maximum value of Equation 1 is one.

$$
\max f(o b s, c o r)=\frac{1}{1+\frac{\left|S^{2} o b s^{-S^{2} c o r \mid}\right|+c * \mid \bar{x}_{o b s} \bar{x}^{-\bar{x}_{c o r} \mid}}{2}}
$$

Cor is corrected data and obs is observation data, $\mathrm{c}$ is any constants to weight the difference of mean, since the value is much smaller than thedifference of variance. This study uses $\mathrm{c}$ of 3 . Binary chromosome, roulette wheel selection, one point crossover, and binary bit flip mutation are used on performing SOGA.

\subsection{Non-dominated Sorting Genetic Algorithm II}

NSGA-II was introduced by Debet al.[13], NSGA-II generates Pareto optimal solution which is a nondominated solution in the criterion space or an optimal solution in the decision space [14]. NSGA-II which is used in this study refers to [15]. However, post process is addedto mitigate production of local maximum solution and to select single solution. In the beginning, NSGA-II is repeated for five times, on each repetition, a set of Pareto solution is stored. Furthermore, solutions of each repetition are combined into one set Pareto solution. The last step, we perform non-dominated sorting to the combinedPareto solution, solutions whose place on the first ranking are selected as final set Pareto solution. Figure 2 shows the process of NSGA-II with additionalpost process. Single solution is selected from set Pareto solution based on criteria of minimal root mean square error (RMSE) of monthly precipitation.

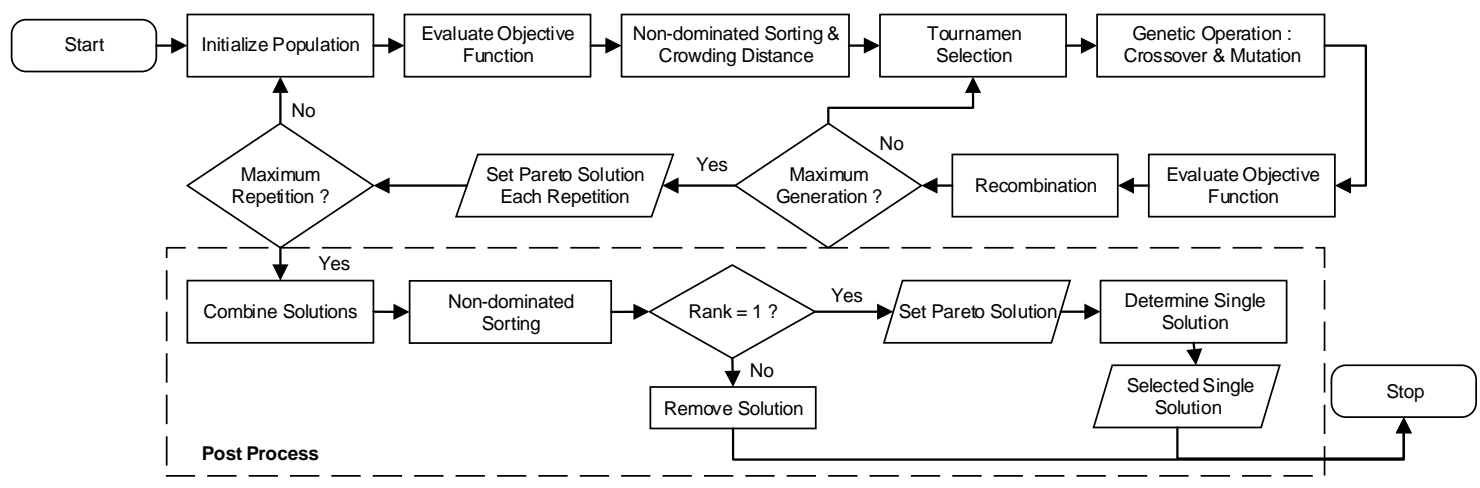

Figure 2. Process of NSGA-II with post process 
Two point crossover and binary bit flip mutation are used for performing NSGA-II. The Objectives of NSGA-II refer to the objectives of estimatingof NL method parameters used by previous studies. The objectives of NSGA-II shown by Equation 2 and 3.

$$
\begin{aligned}
& \max f_{1}(\text { obs }, \text { cor })=\frac{1}{1+\left|\bar{x}_{o b s}-\bar{x}_{c o r}\right|} \\
& \max f_{2}(o b s, c o r)=\frac{1}{1+\left|C V_{o b s}-C V_{c o r}\right|}
\end{aligned}
$$

\subsection{Evaluation}

Evaluation on this research is evaluating the obtained results based on statistical moment and quantile value. Furthermore, corrected CHIRP constructed is compared by using SOGA and NSGA-II, CHIRP and CHIRPS. In addition, evaluation is applied on different month.

\section{Result and Analysis}

\subsection{Proposed Scheme and Search Space of Genetic Algorithm}

Proposed scheme with additional threshold on NL method is showed by Equation 4. Search space is obtained from several experiments by raising and lowering the upper and lower bounds. Search space of $a_{1}$ and $b_{1}$ on Equation 4 are determined such that the parameters are able to reach higher precipitation values of corrected CHIRP. The parameters were expected to address limitation of CHIRP in estimating extreme precipitation. The idea is to transform the high precipitation of CHIRP to extreme precipitation. High precipitation value of CHIRP is represented by $\theta_{1}$, where value of $\theta_{1}$ was around $\bar{x}_{\text {CHIRP }}+s_{C H I R P}$ and $\bar{x}_{C H I R P}+2 s_{C H I R P}$. Upper bound of the parameter $a_{1}$ and $b_{1}$ is determined such that the increased precipitation values are not too extreme.Parameter $a_{2}$ and $b_{2}$ are expected to lower precipitation values around median value of CHIRP.0.1 and 1 as lower and upper bounds of $a_{2}$ and $b_{2}$ are selected since the values could achieve the objective. Based preliminary study, observation data contained many days with no precipitation $(P=0)$. In other hand, CHIRP did not represent it effectively. Hence, we transformed low precipitation of CHIRP to no precipitation. Low precipitation value are represented by $\theta_{3}$, where value of $\theta_{3}$ is around first quantile of CHIRP.

$$
P^{*}=\left\{\begin{array}{c}
a_{1} \cdot P^{b_{1}}, P>\theta_{1} \\
P \quad, \quad \text { otherwise } \\
a_{2} \cdot P^{b_{2}}, 2 \theta_{3}<P<\theta_{2} \text { or } \theta_{2}<P<2 \theta_{3} \\
0, \quad P<\theta_{3}
\end{array}\right.
$$

Subject to,

$$
\begin{aligned}
& \theta_{1}>\theta_{2}>\theta_{3} \\
& \bar{x}_{\text {CHIRP }}+s_{\text {CHIRP }}-2<\theta_{1}<\bar{x}_{\text {CHIRP }}+2 * s_{\text {CHIRP }}+2 \\
& \left(Q_{1_{\text {CHIRP }}} * 2\right)<\theta_{2}<\left(Q_{2} \text { CHIRP }+3\right) \\
& \left(Q_{1_{\text {CHIRP }}} / 2\right)<\theta_{3}<\left(Q_{1_{\text {CHIRP }}}+1\right) \\
& 0.1<a_{2}<1 \\
& 0.1<b_{2}<1 \\
& 0.7<a_{1}<4 \\
& 0.7<b_{1}<4
\end{aligned}
$$

According to the testing process, Equation 4 effectively reduced bias when the following conditions areadequate : (1) Median of CHIRP was between $2 \theta_{3}$ and $\theta_{2}$, and (2) Value of $\theta_{3}$ was slightly higher than first quantile of CHIRP. Figure 3 shows ilustration of bias correction of the scheme. 


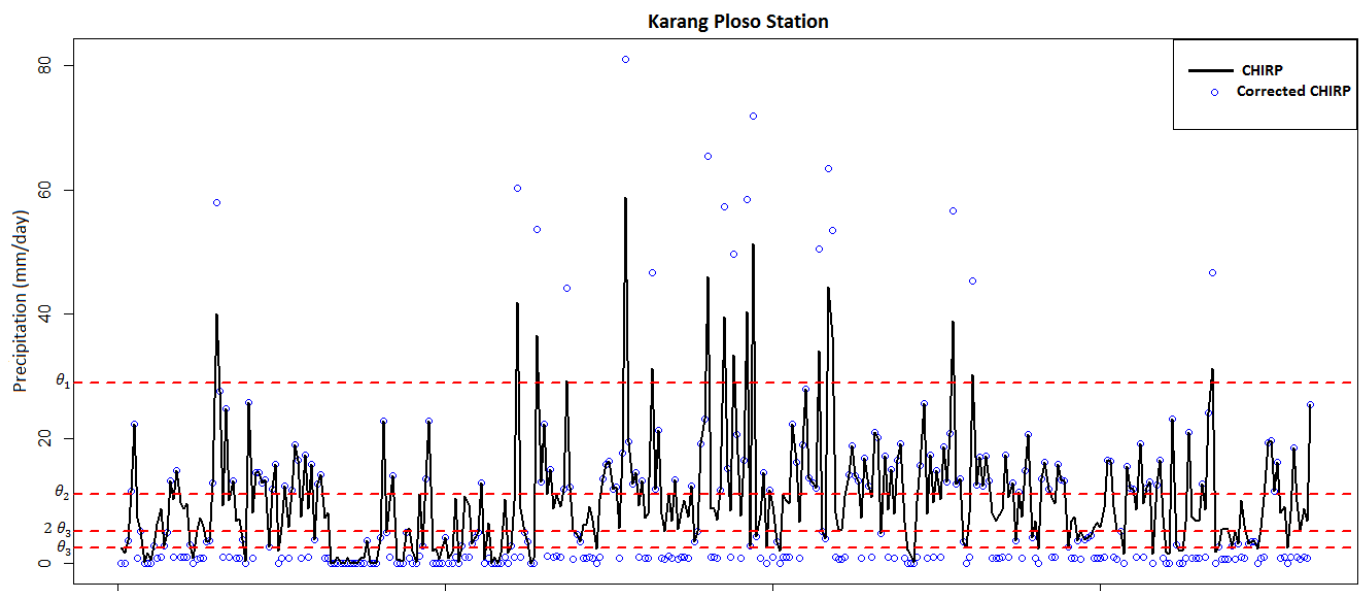

Figure 3. CHIRP and Corrected CHIRP in January

Figure 3 shows that after correction process, a number of data decreased close to zero. The reduction is due to low values of $a_{2}$ and $b_{2}$ produced by GA. The impact of bias correction on data distribution was revealed by Figure 4, and the impacts on stastistical moment and quantile values are spesifically showed by Tabel 1 .

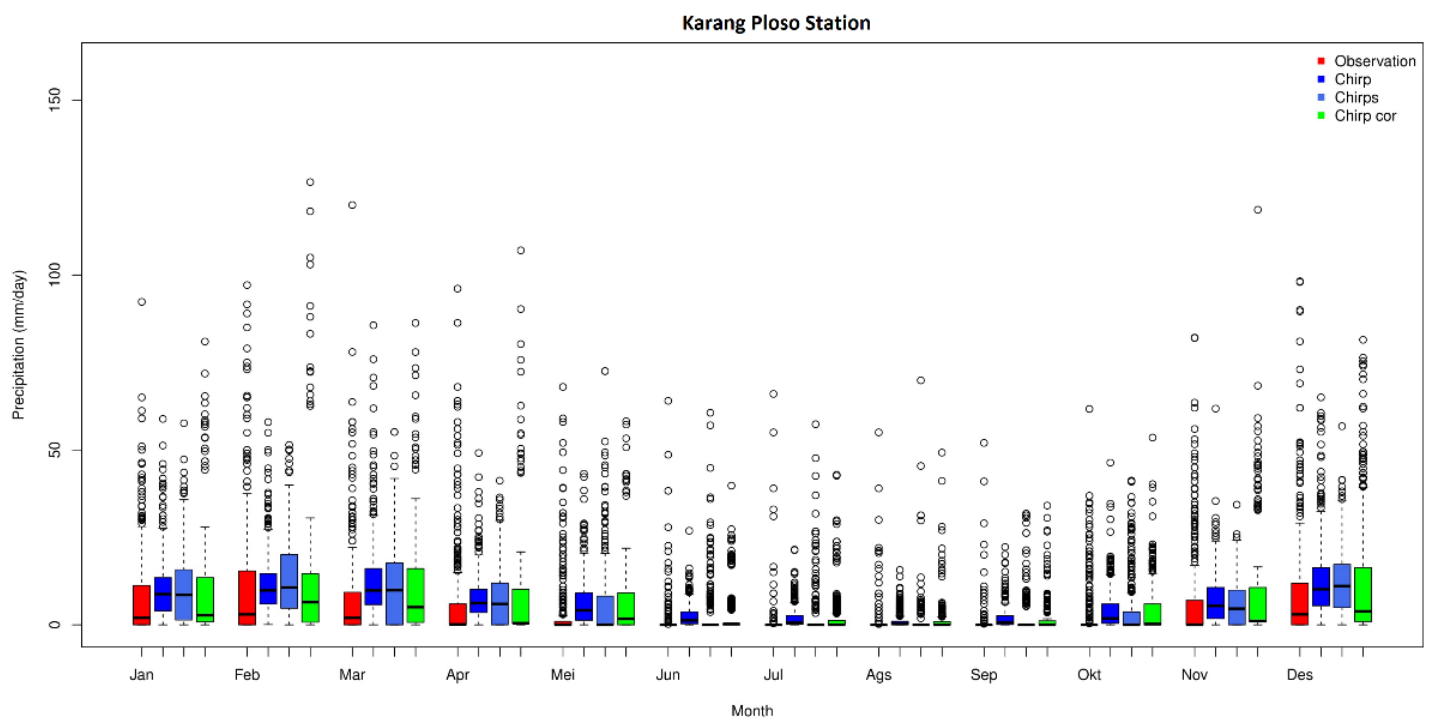

Figure 4. Boxplot Data at Karang Ploso Station

Tabel 1. Statistical Moment and Quantile Values at Karang Ploso Station

\begin{tabular}{|c|c|c|c|c|c|c|c|c|}
\hline & Data & 1st Qu. & Median & 3rd Qu. & Mean & Variance & Skewness & Kurtosis \\
\hline \multirow{4}{*}{ 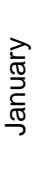 } & Observation & 0 & 2.1 & 11.52 & 8.357 & 171.915 & 2.355 & 6.99 \\
\hline & CHIRP & 4.092 & 8.779 & 13.52 & 10.479 & 80.782 & 1.862 & 5.041 \\
\hline & CHIRPS & 2.752 & 8.632 & 15.8 & 10.505 & 93.502 & 1.263 & 2.375 \\
\hline & CHIRP cor & 0.826 & 2.758 & 13.52 & 8.729 & 170.772 & 2.601 & 8.083 \\
\hline \multirow{4}{*}{ 亏े } & Observation & 0 & 0 & 0 & 0.903 & 31.667 & 8.614 & 81.822 \\
\hline & CHIRP & 0.082 & 0.639 & 2.657 & 2.039 & 9.889 & 2.739 & 9.873 \\
\hline & CHIRPS & 0 & 0 & 0 & 2.036 & 47.161 & 4.503 & 23.737 \\
\hline & CHIRP cor & 0 & 0 & 1.275 & 2.264 & 31.665 & 4.216 & 20.727 \\
\hline
\end{tabular}


Tabel 1 shows that GA sucessfully corrects the variance value both during dry and wet months, this result is also found on other dry and wet months (not shown). First and second quantile are also succesfully corrected. However, third quantile, skewness (third moment), and kurtosis (fourth moment) cannot be corrected robustly. Figure 4 reveals proposed scheme is able to reduce bias on first and second quantiles robustly for all months.

\subsection{Single-objective Genetic Algorithm}

Optimal parameters of SOGA obtained from training process were probability crossover (pc) 0.75 , probability mutation (pm) 0.01 , population size 15 , and number of generations 500 . Result of SOGA is showed in Tabel 2.

Tabel 2. Fitness Values of Four Stations

\begin{tabular}{ccccc}
\hline & Bandung & Tegal & Sangkapura & Banyuwangi \\
\hline Jan & 0.77 & 0.21 & 0.82 & 0.46 \\
Feb & 0.72 & 0.34 & 0.73 & 0.81 \\
Mar & 0.99 & 0.90 & 0.43 & 0.21 \\
Apr & 0.88 & 0.31 & 0.64 & 0.99 \\
May & 0.26 & 0.54 & 0.25 & 0.57 \\
Jun & 0.82 & 0.49 & 0.56 & 0.95 \\
Jul & 0.99 & 0.66 & 0.45 & 0.68 \\
Aug & 0.66 & 0.52 & 0.99 & 0.99 \\
Sep & 0.82 & 0.51 & 0.46 & 0.68 \\
Oct & 0.29 & 0.49 & 0.66 & 0.40 \\
Nov & 0.80 & 0.44 & 0.34 & 0.67 \\
Dec & 0.69 & 0.39 & 0.83 & 0.47 \\
\hline
\end{tabular}

High fitness value reveals SOGA is suitable to estimate paremeter of NL method. Table 2 shows that many months contain low fitness value. Low fitness value indicatsthat the case (equation) cannot be solved using single objective, so thatit is rather be solved using multiobjective optimization. Besides, bias correction process using SOGA leads to increase of monthly precipitation bias as shown in Table 4.

\subsection{Non-dominated Sorting Genetic Algorithm II}

Parameter of NSGA-II was obtained from training process. The parameters were pc 0.7 , pm 0.1 , population size 60 , and number of generations 600 . To reduce the impact of increasing bias on monthly precipitation, We considered time series bias indicator by selecting single solution which yielded low bias on monthly precipitation. Figure 5 is plot of fitness solution yielded by NSGA-II. However, Application of NSGA-II is not invariably yielded Pareto solution.
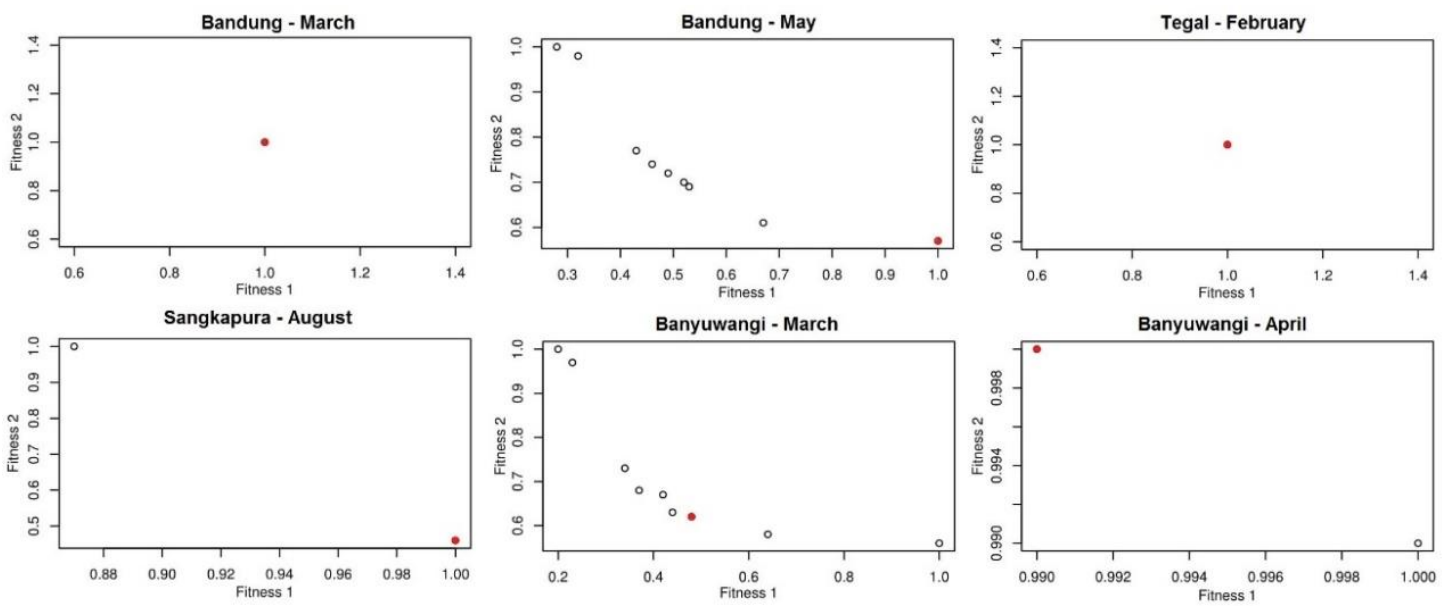

Figure 5. Fitness Solutions of NSGA-II 
Figure 5 reveals NSGA-II yielded Pareto set solution in the time SOGA yields low fitness as shown in Tabel 2. On the other hand, single solution is yielded in the time SOGA yields high fitness value. On Figure 5, this conclution is represented by precipitation in March and May at Bandung Station; March and April at Banyuwangi Station. The conclution is found on other months. However, in February at Tegal Station and in August at Sangkapura Station, the conclution is not valid. In February at Tegal Station, SOGA yields low fitness. It means that single objective is not suitable. However, in the month NSGA-II yields single solution which meanstwo objectivescan be solved without using Pareto princips.

\subsection{Evaluation}

Tabel 3 shows absolute difference of statistical moments and quantile values of data CHIRP, CHIRPS, CHIRP corrected using SOGA and NSGA-II to observation precipitation data.Wet season is represented by December and dry season is represented by Juli. These two months are verified that they are sufficient in representing other months on same season (not shown); December also represents result of January and February; July also represents result of Juny and August. Low value in Tabel 3 means that the difference of data to observation precipitation is low.

Tabel 3. Absolute Difference Data Againts Observation

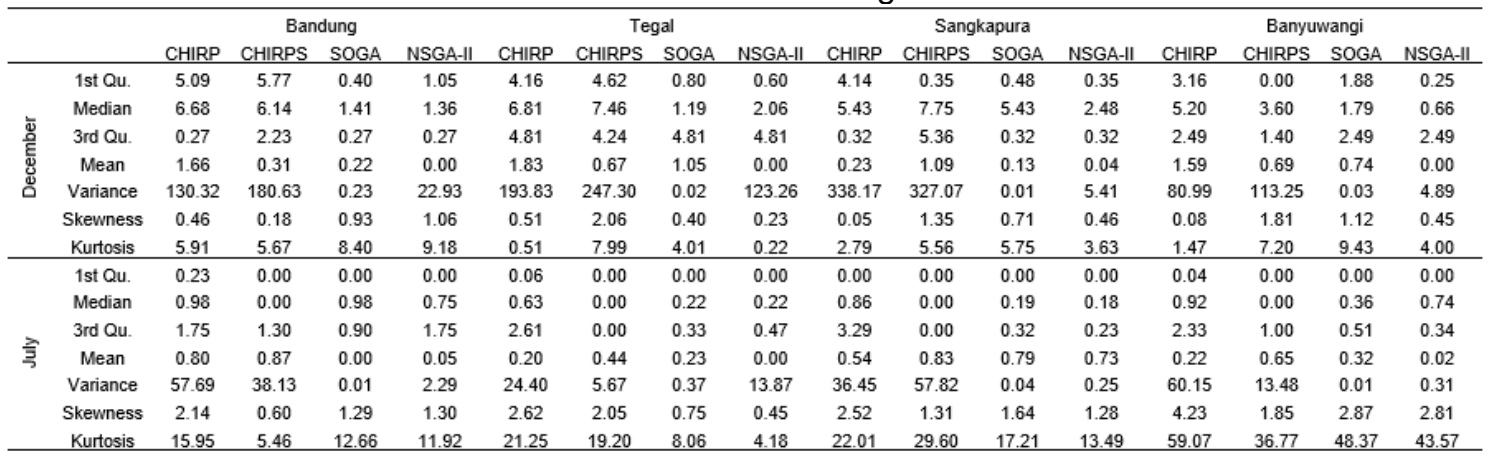

Tabel 3 reveals during dry season, most of bias indicator of CHIRPS out performs CHIRP, including quantile values and variance, however bias on mean is higher. For all stations, first and second quantile bias of CHIRPS are lower than corrected CHIRP during dry season. Both SOGA and NSGA-II robustly reduce bias on first and second quantile and variance duringtwo seasons. However, SOGA cannot robustly reduce bias of CHIRP on mean value during dry season while NSGA-II successfully reduces the bias during two seasons.

During wet season, corrected CHIRP (both SOGA and NSGA-II) robustly outperform CHIRP and CHIRPS for all bias indicators, except third quantile, third and fourth moment. SOGA performs absolutely well in handling bias on variance, it can be seen on Figure 6. Nevertheless, NSGA-II robustly reduces bias on both variance and mean.

Figure 6 represents results of all four stations. Zero value means that quantile value or statistical moment of data matchs to observations. SOGA cannot handle bias on mean value robustly, since the bias in May and October is higher than CHIRP. Moreover, Figure 6 shows that the proposed scheme cannot reduce bias on third quantile. Bias correction process successfully reduces bias on first and second moment. However, bias on third and fourth moment cannot be handled robustly. This results are relevant to [7]. [7] concluded that NL method cannot handle bias on third and fouth moment. In other hand, when data can be approximated using gamma distribution, QMG can robustly reduce bias on third and fourth moment.

Corrected CHIRP obtained using SOGA and NSGA-II both increase bias on monthly precipitation, and lead to decrease of correlation. This results are relevant to [8], NL method is distribution-based bias correction method, based on the characteristics of bias correction, distribution-based method cannot handle bias on time series indicator. Mean-based bias correction methods, including Linear Scalling (LS), Local Intensity Scaling (LOCI) were the best 
method in handling bias on time series indicator [2] [8]. Impact of daily bias correction on monthly precipitation is shown by Tabel 4 .

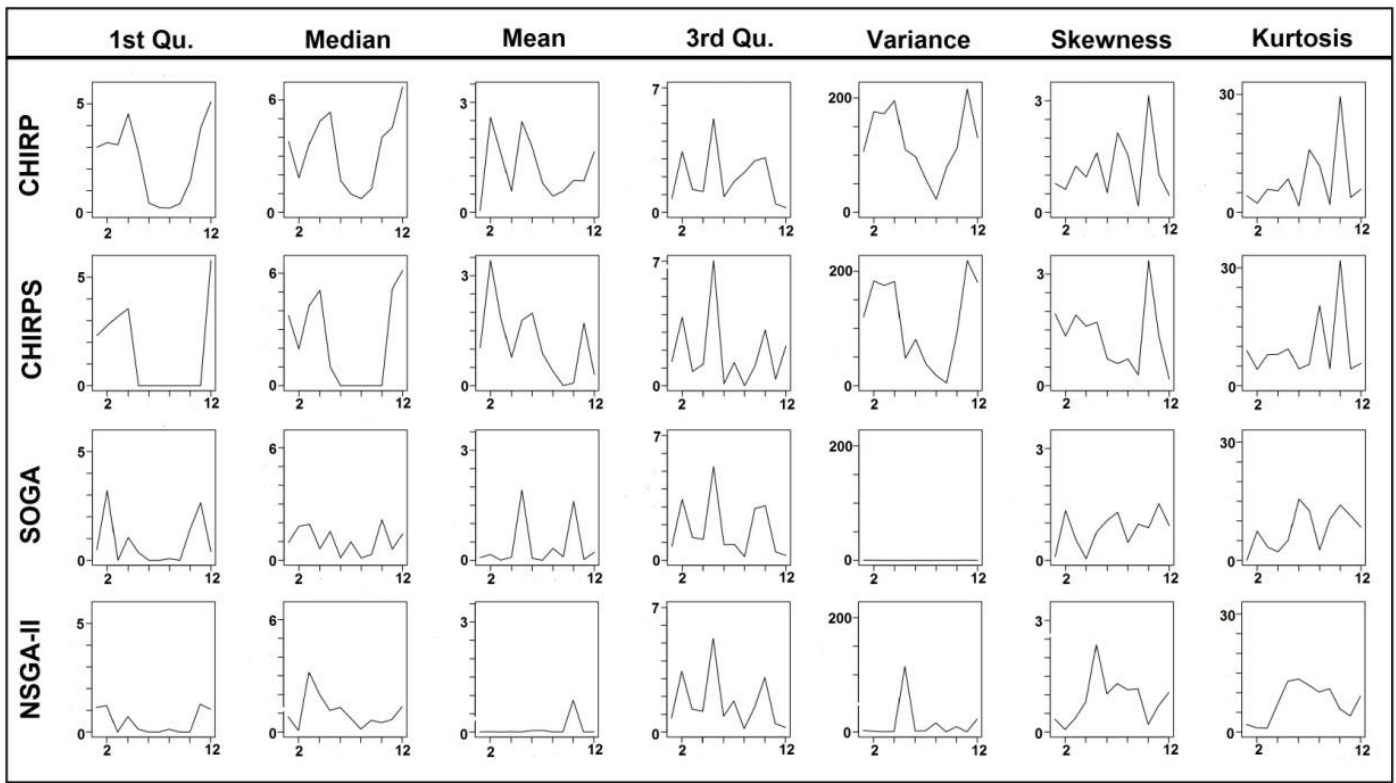

Figure 6. Plot of Absolute Difference Data Againts Obervation at Bandung Station

Tabel 4. Time Series Indicators For Monthly Precipitation

\begin{tabular}{ccccccccc}
\hline \multirow{2}{*}{ Data } & \multicolumn{2}{c}{ Bandung } & \multicolumn{2}{c}{ Tegal } & \multicolumn{2}{c}{ Sangkapura } & \multicolumn{2}{c}{ Banyuwangi } \\
& RMSE & $r$ & RMSE & $r$ & RMSE & $r$ & RMSE & $r$ \\
\hline CHIRP & 104.862 & 0.693 & 89.035 & 0.792 & 80.385 & 0.892 & 72.321 & 0.746 \\
CHIRPS & 105.946 & 0.692 & 69.284 & 0.842 & 69.294 & 0.926 & 59.884 & 0.765 \\
SOGA & 120.22 & 0.671 & 109.887 & 0.709 & 89.194 & 0.883 & 89.573 & 0.708 \\
NSGA-II & 111.608 & 0.705 & 95.472 & 0.727 & 86.411 & 0.886 & 80.901 & 0.708 \\
\hline
\end{tabular}

Tabel 4 reveals application of NSGA-II is able to reduce impact of bias increasing on monthly precipitation than SOGA. Application of NSGA-II has limitation, variance of corrected CHIRP is not ensured absolutely match to observation. As an example, shown in Figure 6 , in May, bias on variance of corrected CHIRP produced using NSGA-II does not decrease significantly. It is an impact of considering time series indicator in selecting single solution from Pareto set.

\section{Conclusion}

The result of this study reveals NSGA-II is suitable to estimate parameter of NL mehod. However, the result is only valid when SOGA yields low fitness value. For condition of high fitness value of SOGA, Pareto concept of NSGA-II is not suitable.Proposed scheme with additional thresholds is able to reduce bias on first and second quantile on certain level. It also robustly reduces bias on first and second quantile. However, Biases on third quantile, third and fourth moment cannot be corrected robustly.

The future study can compare this proposed NL method using NSGA-II to common method used in estimating parameter of NL method, such as Brents and Generalized Reduced Gradient. Clustering process to obtainspatial range of the solution producted by certain station 
is also interesting to conduct. The additional of clustering process can be used to correct all pixels of CHIRP while this study only correctspixels correspondent to station location.

\section{References}

[1] Kim KB, Bray M, Han D. Exploration of optimal time steps for daily precipitation bias correction: a case study using a single grid of RCM on the River Exe in southwest England. Hydrological Sciences Journal. 2016; 61(2): 289-301.

[2] Gumindoga W, Rientjesa THM, Hailec AT, Makurirab H, Reggianid P. Bias correction schemes for $\mathrm{CMORPH}$ satellite rainfall estimates in the Zambezi River Basin. Hydrology and Earth System Sciences. 2016; Discuss.

[3] Pineda RV, Demaría EMC, Valdés JB, Wi S, Capdevila AS. Bias correction of daily satellite-based rainfall estimates for hydrologic forecasting in the Upper Zambezi, Africa. Hydrology and Earth System Sciences. 2016; Discuss.

[4] Funk CC, Peterson PJ, Landsfeld MF, Pedreros DH, Verdin JP, Rowland JD, et al. A quasi-global precipitation time series for drought monitoring. US Geological Survey, 2014; 2327-638X.

[5] Katsanos D, Retalis A, Michaelides S. Validation of a high-resolution precipitation database (CHIRPS) over Cyprus for a 30-year period. Atmospheric Research. 2016; 169: 459-464.

[6] Toté C, Patricio D, Boogaard H, van der Wijngaart R, Tarnavsky E, Funk C. Evaluation of satellite rainfall estimates for drought and flood monitoring in Mozambique. Remote Sensing. 2015; 7(2): 1758-1776.

[7] Lafon T, Dadson S, Buys G, Prudhomme C. Bias correction of daily precipitation simulated by a regional climate model: a comparison of methods. International Journal of Climatology. 2013; 33(6): 1367-1381.

[8] Fang G, Yang J, Chen Y, Zammit C. Comparing bias correction methods in downscaling meteorological variables for a hydrologic impact study in an arid area in China. Hydrology and Earth System Sciences. 2015; 19(6): 2547-2559.

[9] Piani C, Haerter J, Coppola E. Statistical bias correction for daily precipitation in regional climate models over Europe. Theoretical and Applied Climatology. 2010; 99(1-2): 187-192.

[10] Leander R, Buishand TA. Resampling of regional climate model output for the simulation of extreme river flows. Journal of Hydrology. 2007; 332(3): 487-496.

[11] Vernimmen R, Hooijer A, Aldrian E, Van Dijk A. Evaluation and bias correction of satellite rainfall data for drought monitoring in Indonesia. Hydrology and Earth System Sciences. 2012; 16(1): 133.

[12] Zamiri S, Balochian S, Baloochian $\mathrm{H}$. Multi objective optimization of multi component isothermal liquid-phase kinetic sequence using multivariable PI control. Bulletin of Electrical Engineering and Informatics. 2014; 3(4): 277-284

[13] Deb K, Pratap A, Agarwal S, Meyarivan T. A fast and elitist multiobjective genetic algorithm: NSGA-II. IEEE transactions on evolutionary computation. 2002; 6(2): 182-197.

[14] Ratono J, Seminar KB, Arkeman Y, Suroso AI. ERP selection using Fuzzy-MOGA approach: A food enterprise case study. Telkomnika. 2015; 13(3): 1105-1112.

[15] Arkeman Y, Gunawan H, Seminar KB. Algoritma Genetika Teori dan Aplikasinya untuk Bisnis dan Industri. IPB Press; 2012. 